\title{
Dependence of ion channeling on relative atomic number in compounds
}

\author{
K. Nordlund \\ Department of Physics, P. O. Box 43, FIN-00014 University of Helsinki, Finland \\ G. Hobler \\ Institute of Solid State Electronics, TU Wien, Floragasse 7, A-1040 Wien, Austria
}

(Dated: January 17, 2019)

\begin{abstract}
Ion channeling is a well-known radiation effect, important for understanding the passage of energetic ions and recoils in all crystalline materials. Recently we developed molecular dynamics and theory-based approaches to calculate ion channeling systematically over all crystal directions, providing ion 'channeling maps' that easily show in which directions channeling is expected [Nordlund et al., Phys. Rev. B. 94, 214109 (2016)]. As an extension of this work, which only considered pure elements, we consider here ion channeling in compounds. To obtain a comprehensive understanding of the effect of varying atom sizes on ion channeling, we compare compounds with pure elements of the same crystal structure, where the atomic number (nuclear charge) of the element is the average of that of the ions in the compound. The results show that the channeling effects in the compounds are very similar to the corresponding pure element, except if there is a really large $(\gtrsim 10)$ ratio of atomic numbers. The reasons to this are discussed in terms of channeling theory.
\end{abstract}

\section{INTRODUCTION}

Ion channeling is a well-known radiation effect, important for understanding the passage of energetic ions and recoils in all crystalline materials. Remarkably, it was first predicted by atomistic simulations $[1,2]$, one of the first instances of atom-level modelling showing predictive capability. It has been systematically examined by a wide range of experiments (e.g. Refs. [3-6]) as well as analytical theories, binary collision approximation and molecular dynamics computer simulations [2, 5, 7-12] However, there have been very few systematic studies of channeling in a wide angular range outside the principal low-index ones.

Recently we developed a molecular dynamics-based approach to calculate ion channeling systematically over all crystallographically nonequivalent directions, providing ion 'channeling maps' that show immediately in which directions channeling is expected [12]. The results showed that channeling effects can be quite significant even at energies below $1 \mathrm{keV}$, and that in many cases, significant planar channeling occurs also in a wide range of crystal directions between the low-index principal ones. In all the cases studied, a large fraction, $20-60 \%$, of the crystal directions showed channeling. These results have two significant practical implications. First, modern experiments on individual randomly oriented nanostructures will have a large probability of channeling [13, 14]. Second, when ion irradiations are carried out on polycrystalline samples, channeling effects on the results cannot a priori be assumed to be negligible.

In the previous work, we also implemented channeling theory to give the fraction of channeling directions in a manner directly comparable to the simulations [12]. The comparison shows good qualitative agreement. In particular, we found that channeling theory is very good at predicting which channels are active at a given energy, down to sub-keV energies, provided the penetration depth is more than a few atomic layers.

The previous work, however, only considered pure elements. It is a natural question to ask how the channeling (in terms of channel width, penetration depth in the middle of the channel, etc.) is affected by varying atom size in a multi-element system. Experimentally, ion channeling techniques have been widely used to analyze both compound semiconductors [15-19] and metal alloys [20, 21]. However, there have been no systematic theoretical analyses of how atoms in a compound being of unequal size may affect channeling.

To start obtaining an understanding of the effect of varying atom sizes in compounds on ion channeling, in this work we compare compounds of varying relative atomic number (nuclear charge) with pure elements of the same crystal structure. Channeling for a given crystal structure of course depends not only on crystal structure and atom size, but also on lattice constant. To be able to uniquely determine the effect of atom size, we here carry out a model study, where we fix the lattice constant to be the same in the compared compounds. To clearly see the possible effects of varying atomic number, we also include hypothetical crystal structures with the same crystal structure and average atomic number $Z$ as the reference structure, but increasingly large difference in atomic number of the two parts making up the compound. We use the atomic number as the reference since channeling theory depends directly on this quantity [8].

\section{METHOD}

Channeling was for this work simulated with the same approach as introduced in Ref. [12]. As a brief summary, the recoil interaction approximation (RIA) of molecular dynamics (MD) and the MDRANGE code [22-24] were used to simulate ion penetration depths. The surface normal orientation was always the [001] crystal direction, 
and the incoming ion direction was tilted with respect to this. The initial position of the ion was $3 \AA$ outside the sample surface in the $z$ direction, and randomly selected in the $x$ and $y$ directions over a surface area of one or $2 \times 2$ unit cells of the crystal structure.

The ion range was determined as the projected range [25] with respect to the incoming ion direction. To get the channeling over all crystal directions in crystals with cubic symmetry, we scanned over all angles $\theta$ from $0^{\circ}$ to $89^{\circ}$ and $\varphi$ from $0^{\circ}$ to $90^{\circ}$ in $1^{\circ}$ intervals. For sufficient statistics, 1000-3000 ions were simulated for each $(\theta, \varphi)$ pair. For the plotting, bilinear interpolation [26] was used in between the $(\theta, \varphi)$ pairs to get a smooth 'channeling map' plot. Note that for large $\theta$ values, the fraction of reflected ions starts to increase strongly. When there were no implanted ions, the channeling map plots are left empty. The range values at the largest $\theta$ values just before total reflection may have peculiarly large values, since the range is counted for only a very few non-reflected ions which happen to be scattered inwards to the crystal.

Lattice atoms were in all cases given random thermal displacements with the Debye model [27-29] corresponding to $300 \mathrm{~K}$, using in all Ge-related cases a Debye temperature of $360 \mathrm{~K}$ and all NaCl-related cases a $300 \mathrm{~K}$. The displacement calculation is described in detail in Appendix A.

To study the atom size effect in compounds, we chose two systems where there exist an element or compounds with similar crystal structure and average atom number.

The first set of systems was was $\mathrm{NaCl}(Z=11,17)$ and $\operatorname{KF}(Z=19,9)$, which have the same rock salt (simple cubic) crystal structure and average atomic number $Z$, and a similar lattice constant. Since the compound effects on channeling turned out to be very small, we also added two hypothetical crystals with the same structure, chosen such that the average $Z$ remains the same and the lighter atom type rises one row upwards in the periodic table at each step. Thus the hypothetical structure 'CoH' $(Z=27,1)$ was added to the set of system studied. The lattice constant was kept at the $\mathrm{NaCl}$ value in all cases, and the mass of Co was selected such that the average mass of ' $\mathrm{CoH}$ ' is the same as for $\mathrm{NaCl}$. To improve on the understanding of the transformation of the shape of the channeling maps from $\mathrm{NaCl}$ to ' $\mathrm{CoH}$ ', we also created hypothetical crystals of 'Si' where all atoms in $\mathrm{NaCl}$ are replaced with $\mathrm{Si}$ with the same mass as the average of $\mathrm{NaCl}$, and of 'Ni' where $\mathrm{Na}$ is replaced with 'Ni' and the second atom sublattice $(\mathrm{Cl})$ is completely removed. The crystal structure of 'Si' is simple cubic, and the 'Ni' sublattice is a regular face-centered cubic lattice.

The second set of systems Ge and InP, which have essentially the same crystal structure (Ge has the diamond structure, which is the same as the $\mathrm{InP}$ zincblende one if the atom types are equal). Note that both systems have the same average atomic number $(Z=32$ for $\mathrm{Ge}, Z=49,15$ for In and $\mathrm{P}$, respectively) and almost equal atom masses $(m=72.61 u$ for Ge and average $m_{\text {ave }}=72.90 u$ for InP). The lattice constant of real InP is $5.87 \AA$. However, as motivated in the introduction, we used the same lattice as for Ge $(5.66 \AA)$. Similar to the NACl-KF system, two hypothetical structures were added, namely 'BaO' $(Z=56,8)$ and 'EuH' $(Z=63,1)$. To emphasize that we do not expect these compounds to exist in reality with this crystal structure, we mark them in hyphens. In both hypothetical cases, the lattice constant was kept the same as for Ge, and the heavier atom mass was also set to a value which gave the same atom average mass as for Ge.

The $\mathrm{NaCl}$ results are also interesting in that the previous work [12] did not consider any crystal with the simple cubic structure.

For the Ge-related systems, we simulated irradiation with $10 \mathrm{keV}$ Ge ions in Ge, InP, 'BaO' and 'EuH', as well as $10 \mathrm{keV} N$ ions in Ge and InP. For the NaCl-related systems we simulated $1 \mathrm{keV} \mathrm{H}$ irradiation of $\mathrm{NaCl}, \mathrm{KF}$ and ' $\mathrm{CoH}$ ', as well as the elemental case of 'Ni'.

In both sets of simulations, the electronic stopping power was also kept the same as in the reference crystal ( $\mathrm{Ge}$ or $\mathrm{NaCl}$, respectively).

In principle, in a compound also the type of the atoms in the top surface atom layer could affect the range results. Tests of switching the top atom layer (sublattice atom types) in InP showed no statistically significant difference in the results, indicating this is not of significance when the ion penetration depths are much deeper than the atom layer thickness.

\section{RESULTS}

\section{A. NaCl-related systems}

Channeling maps for the NaCl-related systems are shown in Fig. 1. In all cases, the [001] direction clearly shows the strongest channeling, which is natural considering the simple cubic structure of the crystal. The $\langle 110\rangle$ and $\langle 111\rangle$ directions also show strong channeling, while the $\langle 112\rangle$ channels are weaker. There is strong planar $\{100\}$ channeling extending from [001] towards the $\langle 110\rangle$ directions.

The results in Fig. 2 confirm the visual impression of Fig. 1 that $\mathrm{NaCl}$ ( $Z$ ratio 1.54 ) and $\mathrm{KF}$ ( $Z$ ratio 2.11 ) show very similar results. The very artificial ' $\mathrm{CoH}$ ' crystal ( $Z$ ratio 27 ) does show clear differences, showing much deeper penetration in several of the channeling directions.

The channeling of the hypothetical 'Si' simple cubic crystal is practically identical to $\mathrm{NaCl}$. The channeling map of the hypothetical ' $\mathrm{Ni}$ ' with $\mathrm{NaCl}$ lattice constant (Fig. 3) is qualitatively similar as that of ' $\mathrm{CoH}$ ', in particular the planar channeling directions are the same, and clearly different from $\mathrm{NaCl}$. 
a)
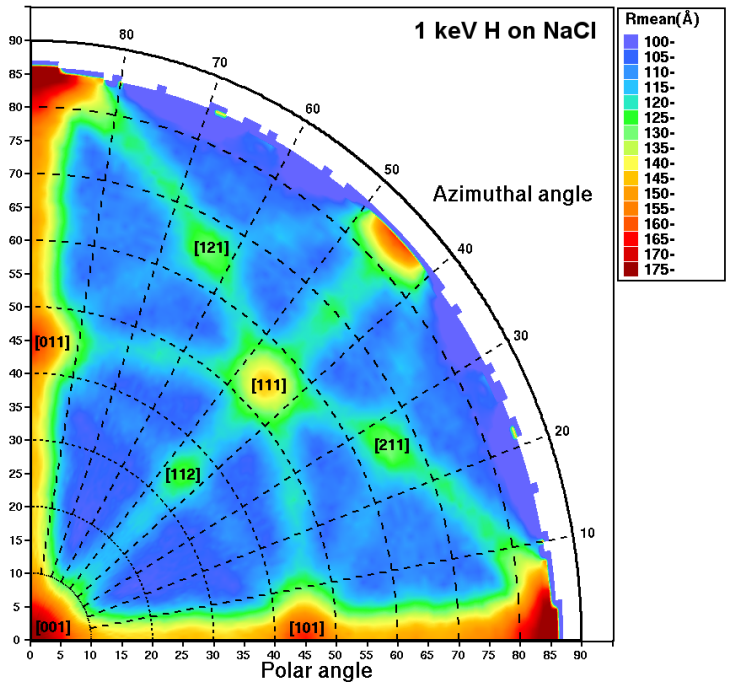

b)
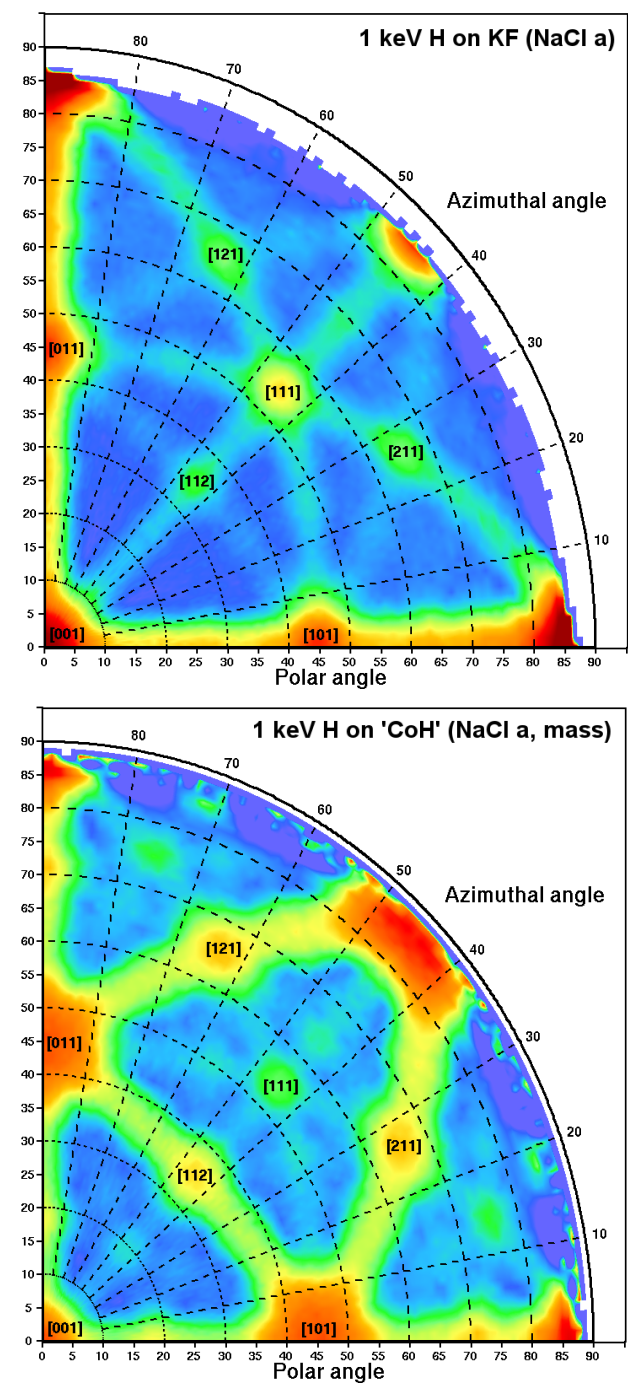

FIG. 1. (Color online) Channeling maps for $1 \mathrm{keV} \mathrm{H}$ ions in a) $\mathrm{NaCl}, \mathrm{b}) \mathrm{KF}$ and c) ' $\mathrm{CoH}$ '. In these cases, the results for the azimuthal angle $\varphi>45^{\circ}$ are reflected by crystal symmetry from the values $\varphi<45^{\circ}$.

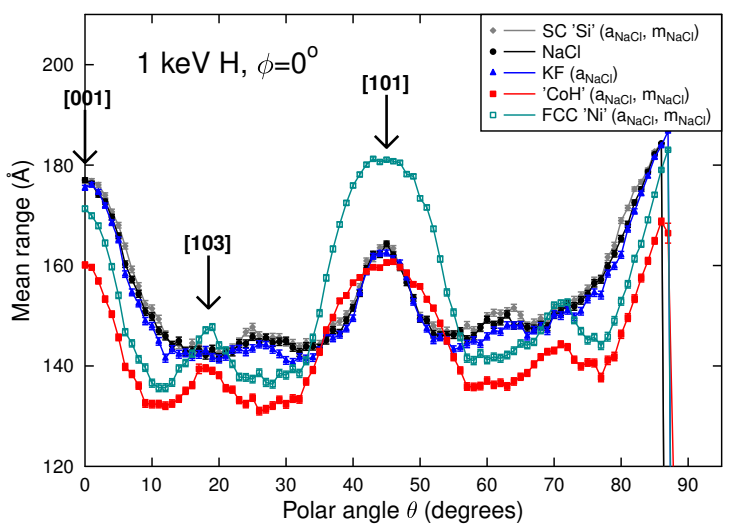

a)

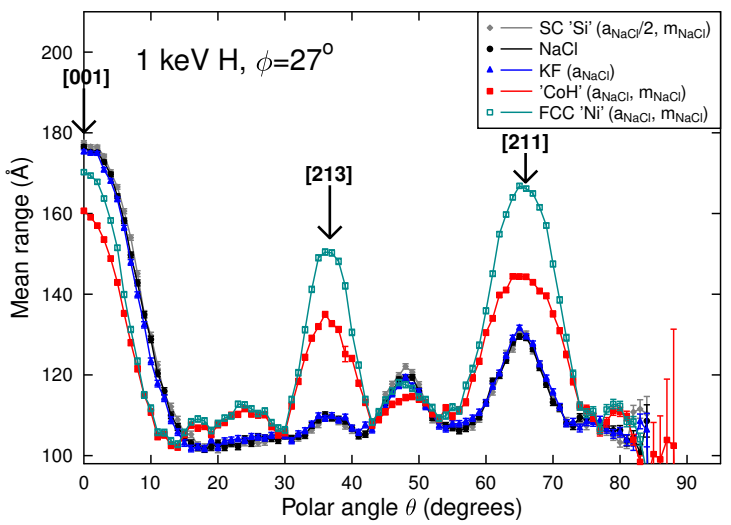

FIG. 2. (Color online) Mean ion ranges of $1 \mathrm{keV} \mathrm{H}$ ions in various rocksalt-structured crystals with the $\mathrm{NaCl}$ crystal structure and lattice constant as a function of the polar (offnormal) angle $\theta$ for a) $\varphi=0^{\circ}$ and b) $\varphi=27^{\circ}$. Also shown are the hypothetical elemental crystals of a simple cubic crystal of $\mathrm{NaCl}$ structure where all atoms are 'Si' with the atom mass same as the average of $\mathrm{NaCl}$, and a crystal of face-centered cubic 'Ni' at atom positions of the $\mathrm{Na}$ sublattice of $\mathrm{NaCl}$. Some of the most important channels are indicated in the figures.

\section{B. Ge-related systems}

Channeling maps for the $10 \mathrm{keV}$ Ge ion irradiation of Ge, 'BaO' and 'EuH' are shown in Fig. 4. The channeling map of Ge in Ge has a qualitatively very similar form as those for $\mathrm{Si}$ in the keV energy range [12], in that the strongest and widest channeling directions are $\langle 110\rangle$, $\langle 211\rangle,\langle 111\rangle$ and $\langle 100\rangle$, in this order.

The case of $\mathrm{Ge}$ on $\mathrm{InP}$ is not shown since the figure is visually indistinguishable from that for the Ge target (Fig. 4a). Also the channeling maps for $10 \mathrm{keV} \mathrm{N}$ ions on Ge and InP gave practically identical results (not shown). Even the compound map for 'BaO' is remarkably similar to that of $\mathrm{Ge}$, even though the ratio between the atomic numbers $Z$ of $\mathrm{Ba}$ and $\mathrm{O}$ is a factor of 7. Only the ' $\mathrm{EuH}$ ' map shows clear differences to the Ge map. 


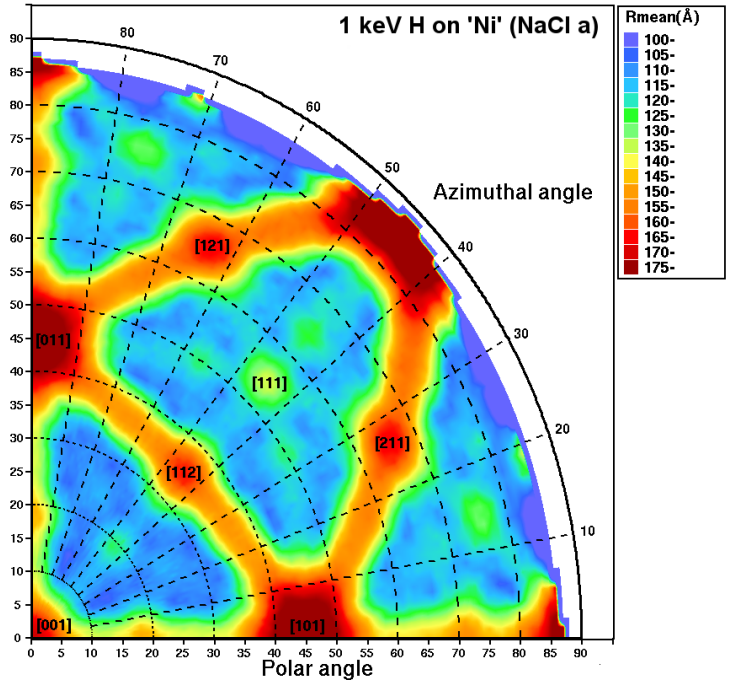

FIG. 3. (Color online) Channeling maps for $1 \mathrm{keV} \mathrm{H}$ ions in hypothetical face-centered cubic 'Ni' with the same lattice constant as the $\mathrm{Na}$ sublattice in $\mathrm{NaCl}$.

A quantitative comparison of channeling in the Ge systems is given for $\varphi=0^{\circ}$ and $\varphi=27^{\circ}$ in Fig. 5. The two azimuthal (twist) angles $\varphi$ were chosen to compare channeling through the strong $\langle 110\rangle$ channel $\left(\varphi=0^{\circ}\right.$, $\left.\theta=45^{\circ}\right)$ with other weaker channeling regions $\left(\varphi=27^{\circ}\right)$. The results show that the Ge and InP results are indeed very similar. The 'BaO' does have a statistically significant difference, in particular showing stronger channeling around the [211] direction. The very artificial case of 'EuH', with a $Z$ ratio of 63 , does show clearly different channeling, even showing extra channeling peaks at e.g. $\varphi=0^{\circ}, \theta \approx 20^{\circ}$ ([103] channel) that are barely visible in $\mathrm{Ge}, \mathrm{InP}$ and $\mathrm{BaO}$. These peaks are also visible in pure FCC metals such as $\mathrm{Au}$ [12]. This indicates that the $\mathrm{H}$ atoms are so small that the channeling is dominated by the FCC Eu sublattice.

From this observation, and that of the ' $\mathrm{CoH}$ ' map given above, we conclude that in the case of very large mass differences in a compound, the channeling features shift towards those in the sublattice of the higher $Z$ atom type.

\section{DISCUSSION}

The small differences between channeling in pure elements and compounds can be understood in terms of channeling theory. In the theory [8], the motion of an ion is described by a continuum potential $U\left(=U_{\mathrm{R}}(\vec{x})\right.$ and $U_{\mathrm{P}}(\vec{x})$ for axial and planar channeling, respectively). $U$ is given by the sum of the continuum potentials of the nearby atomic rows or planes. For the Ziegler-BiersackLittmark (ZBL) interatomic potential [30], the single-row

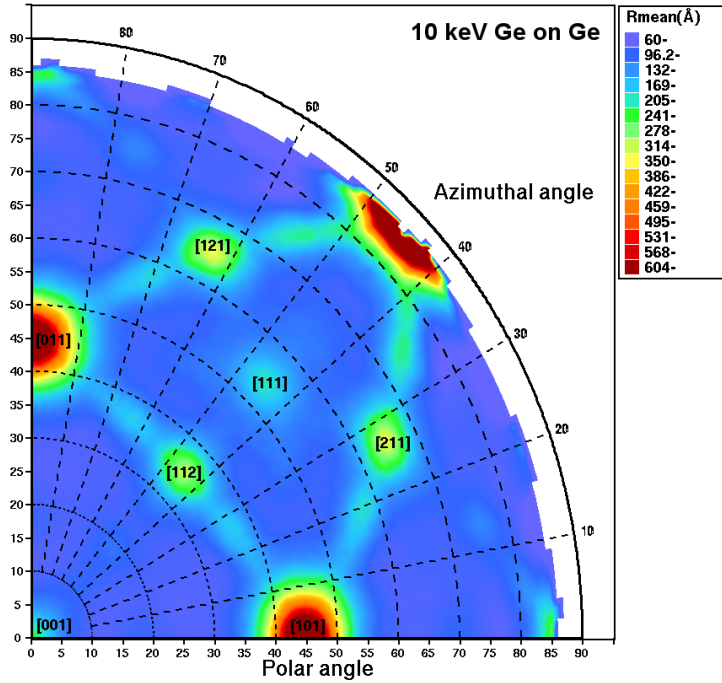

a)

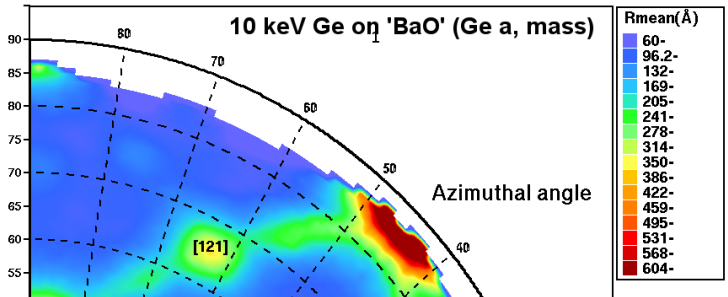

b)

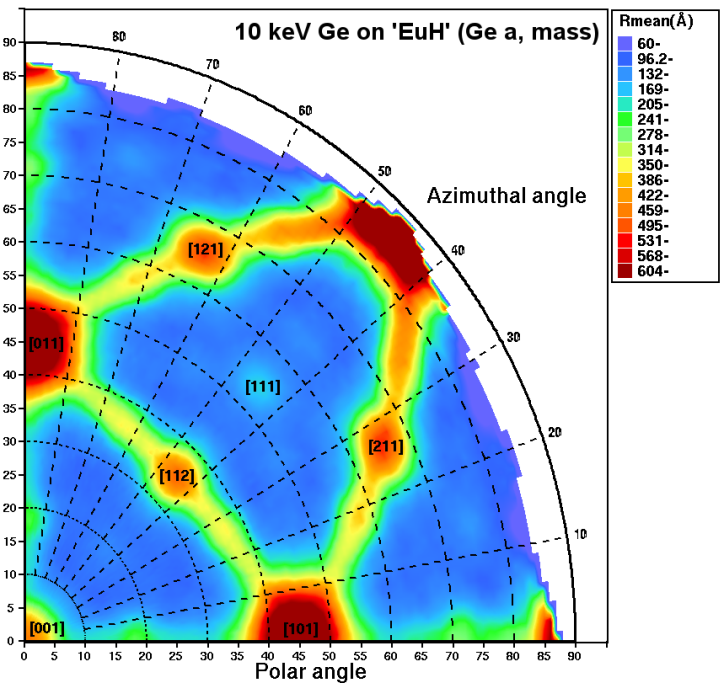

FIG. 4. (Color online) Channeling maps for $10 \mathrm{keV}$ Ge in a) $\mathrm{Ge}, \mathrm{b})$ ' $\mathrm{BaO}$ ' and c) 'EuH'. The map for Ge in InP is visually identical to that for Ge in Ge and hence is not shown. 


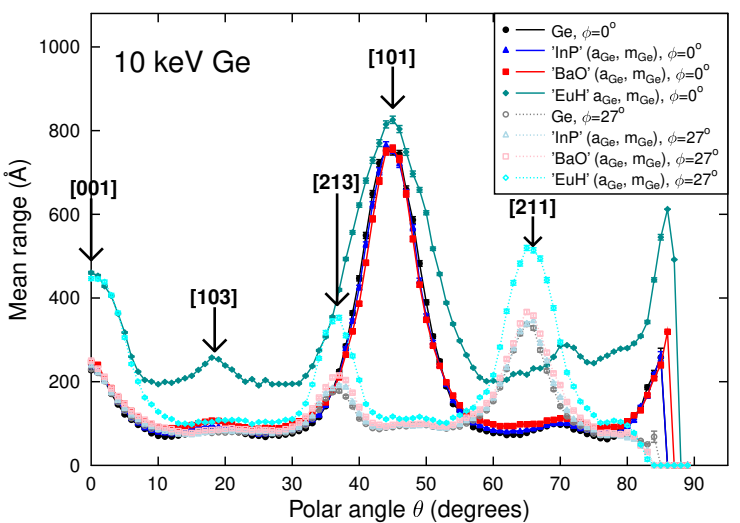

FIG. 5. (Color online) Mean ion ranges of $10 \mathrm{keV}$ Ge ions in various crystals with the Ge crystal structure and lattice constant as a function of the polar (off-normal) angle $\theta$ for $\varphi=0^{\circ}$ and $\varphi=27^{\circ}$. Some of the most important channels are also indicated in the figure.

and single-plane continuum potentials read

$$
U_{\mathrm{R} 1}(r)=\frac{2 Z_{1} Z_{2} e^{2}}{\bar{d}_{\mathrm{R}}} \sum_{i=1}^{4} a_{i} \mathrm{~K}_{0}\left(\frac{b_{i} r}{a_{\mathrm{ZBL}}}\right)
$$

and

$$
U_{\mathrm{P} 1}(r)=2 \pi N_{2} Z_{1} Z_{2} e^{2} a_{\mathrm{ZBL}} \sum_{i=1}^{4} \frac{a_{i}}{b_{i}} \exp \left(-\frac{b_{i} r}{a_{\mathrm{ZBL}}}\right),
$$

respectively. Here $Z_{1}$ is the atomic number of the ion, $Z_{2}$ of the target atoms, $a_{i}$ and $b_{i}$ are screening function constants (that are the same for all materials) of the universal ZBL interatomic potential [30], $\bar{d}_{\mathrm{R}}$ is the mean distance of the atoms in the row and $N_{2}$ is the areal density of atoms in the planes. The quantity $a_{\mathrm{ZBL}}$ is the ZBL screening length,

$$
a_{\mathrm{ZBL}}=\frac{0.8854 a_{0}}{Z_{1}^{0.23}+Z_{2}^{0.23}}
$$

where $a_{0}$ is the Bohr atomic radius, $0.529 \AA$.

Figure 6 shows the axial continuum potential $U_{\mathrm{R}}(\vec{x})$ for Ge ions in two channels of InP (solid lines) and Ge (dotted lines). [110] in a zincblende structure represents a direction where there exist two kinds of rows, each containing a single atom type. In Fig. 6 the upper left, lower left, and far right atom rows, indicated by the black dots, represent rows of the more strongly interacting In, while the other rows contain $\mathrm{P}$ atoms. [111], on the other hand, represents a direction where all atom rows contain both atom species. For the former, the continuum potential $U_{\mathrm{R} 1}$ is simply obtained by using the appropriate atomic number $Z_{2}$ in Eq. 1 , while for the latter $U_{\mathrm{R} 1}$ is given by the average of the $U_{\mathrm{R} 1}$ for each $Z_{2}$.

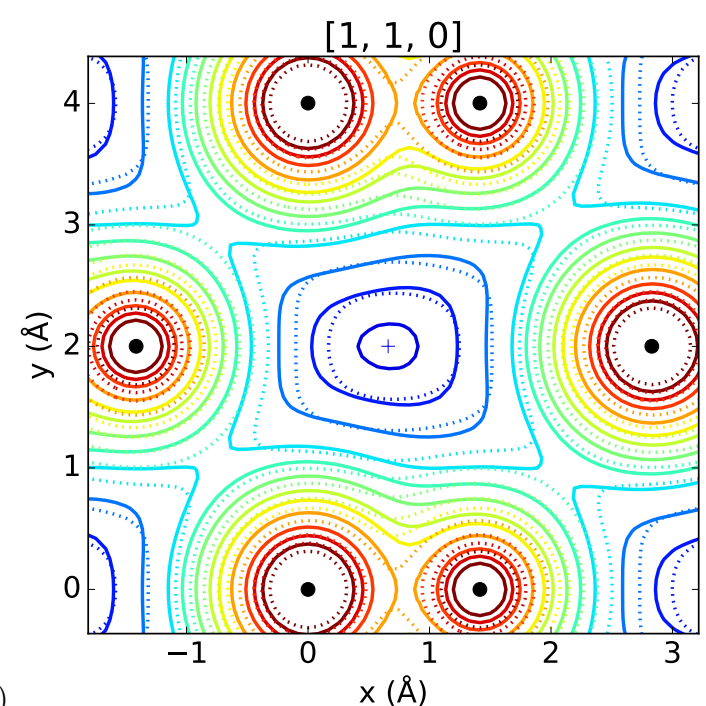

a)

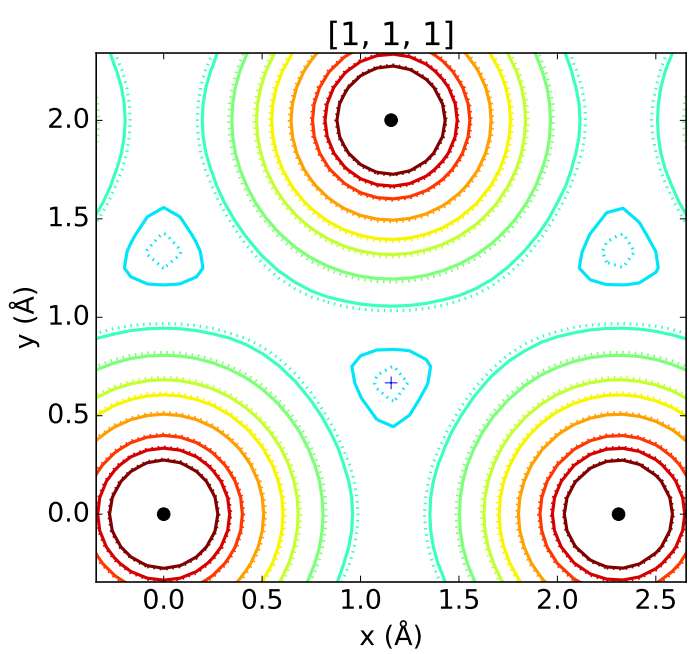

FIG. 6. (Color online) Continuum potentials $U_{\mathrm{R}}(\vec{x})$ for $\mathrm{Ge}$ in InP (solid lines) and Ge in Ge (dotted lines) in a) the [110] channel and b) the [111] channel. Black dots mark the positions of the atom rows. The contour lines range from $15 \mathrm{eV}$ (dark blue) to $1000 \mathrm{eV}$ (dark red) with approximately equally distributed on a logarithmic scale. The crosses mark the energy minima.

To understand the relative insensitivity of channeling to the atom size in compounds, it is important to notice that the average of the continuum potentials for two atomic numbers $Z_{2}$ is close to the continuum potential of the average atomic number, if they are not too different. Both expressions for the continuum potential $U$, Eqs. 1 or 2 , have a prefactor that is linear with $Z_{2}$, so the averaged continuum potential prefactor will simply depend on the average of $Z_{2}$ over the two atom types. The screening length $a_{\mathrm{ZBL}}$ does also depend on $Z_{2}$ and in a nonlinear manner, i.e., averaging of the whole term of Eq. 1 or 2, does not exactly give a linear average in $Z$. However, the dependence of $a_{\mathrm{ZBL}}$ on $Z_{2}$ is weak, via the term $Z_{1}^{0.23}+Z_{2}^{0.23}$. 
In the following, we assume that the dependence of $a_{\mathrm{ZBL}}$ on $Z_{2}$ can be neglected. Then for channels that mix the atom types in their rows such as the zincblende [111] direction (Fig. 6b), the continuum potential of the compound is equal to the continuum potential of the pure element with the average atomic number. In addition, one has to consider the critical approach distance which, for the axial case, can be calculated from [8]

$$
4 E / \hat{d}_{\mathrm{R}}^{2}=\left[\frac{U_{\mathrm{R} 1}^{\prime \prime}\left(r_{\text {crit }}\right)}{1.15 U_{\mathrm{R} 1}^{\prime}\left(r_{\text {crit }}\right)}\right]^{2} U_{\mathrm{R} 1}\left(r_{\text {crit }}\right),
$$

and the critical angle for channeling

$$
\psi_{\text {crit }}=\sqrt{\frac{U\left(r_{\text {crit }}\right)-U_{\text {min }}}{E}} .
$$

$E$ denotes the ion energy and $\hat{d}_{\mathrm{R}}$ the maximum distance between atoms in the row (which differs from the mean distance $\bar{d}_{\mathrm{R}}$ in case of unequal spacing). From Eq. 4 it can be seen that $U_{\mathrm{R} 1}\left(r_{\text {crit }}\right)$ is independent of $Z_{2}$, since the prefactor of Eq. 1 cancels in the term in square brackets in Eq. 4. The potential minimum $U_{\min }$ (at the points marked by crosses in Fig. 6) is equal for the compound and the pure element with average $Z$, and so is the critical angle $\psi_{\text {crit }}$.

For the other kind of channel, where rows are always composed of one atom kind, the continuum potential differs even when the dependence of $a_{\mathrm{ZBL}}$ on $Z_{2}$ can be neglected, see Fig. 6a. However, if the potential minimum is a point of high enough symmetry such as in Fig. 6a, $U_{\text {min }}$ can be considered to be composed of pairs of singlerow potentials of equally distant rows of different atom type. Since the sum of two values is twice their mean and the independence of $U_{\mathrm{R} 1}\left(r_{\text {crit }}\right)$ from $Z_{2}$ holds, $\psi_{\text {crit }}$ still only depends on the average of $Z_{2}$ also for the second kind of channel.

We have silently assumed that $U_{\mathrm{R}}\left(r_{\text {crit }}\right)=U_{\mathrm{R} 1}\left(r_{\text {crit }}\right)$, which is well fulfilled at high energies where $U_{\mathrm{R} 1}\left(r_{\text {crit }}\right)$ is high and $r_{\text {crit }}$ is small, so the influence of neighboring rows can be neglected at $r=r_{\text {crit }}$ from a row. When the ion energy is lowered, $r_{\text {crit }}$ becomes larger, and the approximation becomes less valid. In particular, when $r_{\text {crit }}$ reaches the distance between the channel center and the atomic rows, channeling ceases to be possible which defines the low-energy limit to channeling for the respective channel. Close to this limit, the channeling characteristics are expected to not only depend on the average $Z_{2}$. This can also be seen from the fact that while $U_{\mathrm{R} 1}\left(r_{\text {crit }}\right)$ is independent of $Z_{2}$, this is not true of $r_{\text {crit }}$, since the prefactor of $U_{\mathrm{R} 1}(r)$ contains $Z_{2}$. Therefore, there should be an energy range for each channel of the second kind, where channeling in the pure element is possible while this is not so in the compound. We do not observe this in our MD simulations, so we conclude that it is an unlikely effect.

Channeling theory could certainly be improved for compounds. For channels where the atom rows contain only a single atom type each, like [110] in zincblende
(Fig. 6a), dechanneling is dominated by the more strongly interacting atom type, which controls the critical radius and thus the minimum energy for channeling. For rows that contain both atom species (Fig. 6b), the criterion for determining $r_{\text {crit }}$ (Eq. 4) would need to be modified. Simulations similar to the ones carried out here could be used to test such an extension. A mathematically rigorous channeling theory for compound systems, however, is beyond the scope of the present Article.

Nevertheless, channeling theory provides a reasonable explanation to why channeling of compounds is very similar to the corresponding pure element, except when there is a large ratio of the target atomic numbers $Z_{2}$. Also, since $a_{\mathrm{ZBL}}$ depends on $Z_{1}^{0.23}+Z_{2}^{0.23}$, deviations between compound and pure element are more likely to be observed for light ions. In this respect, the $\mathrm{H}$ bombardments shown in Figs. 1 and 2 represent a worst case.

Considering possible non-model systems, there can of course be other compound effects on channeling. When the lattice constant changes, the interrow and interplanar spacings will, and hence the channeling will also change in particular due to a change in $U_{\min }$ and the minimum energy for channeling. Moreover, the electronic stopping is weaker in channeling directions [23], which could lead to differences in the ion ranges in channels (even though according to channeling theory, the angular channel widths should not change). Finally, for very low energies, when ions move only a few atomic layers, the concept of a continuum potential breaks down, and one might observe larger compound effects - although it is doubtful whether these should be called channeling.

\section{CONCLUSIONS}

The results presented in this work show that the channeling effects in compounds are, at least in the keV energy range, very similar to a corresponding pure element of the same crystal structure and average atomic number (nuclear charge) $Z$. The differences become strong only when the ratio between atomic numbers $Z$ exceeds $\sim 10$. These results imply that channeling simulations or theory for a pure element can be used as a first approximation also for compounds and alloys.

\section{APPENDIX A. METHODS TO ESTIMATE TEMPERATURE DEPENDENCE OF ION CHANNELING}

The thermal displacements of atoms around their equilibrium positions can affect the ion channeling [28]. This effect can be esimated from the Debye model. The one-dimensional root-mean-square displacement amplitude can be obtained using $[27,28]$

$$
u_{i}=\sqrt{\frac{3 \hbar^{2}}{k_{B}}} \sqrt{\frac{\frac{\Phi(x)}{x}+\frac{1}{4}}{m T_{D}}}
$$


where $i$ denotes the $x, y$, or $z$ direction, $x=T_{D} / T, T$ is the sample temperature, $m$ the mass of the sample atoms, and $T_{D}$ the Debye temperature of the material. If $m$ is given in amu's, the constants can be calculated to give

$$
u_{i}=12.063 \sqrt{\frac{\frac{\Phi(x)}{x}+\frac{1}{4}}{m T_{D}}} \AA
$$

$\Phi(x)$ is the Debye integral

$$
\Phi(x)=\frac{1}{x} \int_{0}^{x} \frac{\xi d \xi}{e^{\xi}-1}
$$

This integral cannot be done in closed form, but can be readily integrated numerically. In MDRANGE we implement the displacements using one of two numerical approximations.

An analytically solvable approximation of the integral (8) can be obtained as follows. If $x=T_{D} / T$ is not too large (i.e. $T \gtrsim T_{D}$ ), the exponential in the integral can be approximated with the first three terms of its Taylor series. Then one obtains

$$
\begin{aligned}
\Phi(x) & \approx \frac{1}{x} \int_{0}^{x} \frac{\xi d \xi}{1+\xi+\frac{1}{2} \xi^{2}-1} \\
& =\frac{2}{x} \log \left(1+\frac{x}{2}\right) \\
& \approx \frac{2}{x}\left(\frac{x}{2}-\frac{1}{2} \frac{x^{2}}{4}\right)=1-\frac{1}{4} x
\end{aligned}
$$

where in the last stage $\log (1+x)$ was approximated with the first two terms of its Taylor series. This approximation is compared with the integral evaluated accurately numerically in the inset of Fig. 7. Inserting this into Eq. (6) one obtains a simple expression for the displacements valid at high temperatures:

$$
u_{i}=\sqrt{\frac{3 \hbar^{2}}{k_{B}}} \frac{\sqrt{\frac{T}{m}}}{T_{D}}
$$

Note that this form predicts $u_{i}=0$ at $0 \mathrm{~K}$, which is not consistent with the existence of quantum mechanical zero-point vibrations [31]. However, it can be useful for model studies of channeling effects in the complete absence of thermal vibrations.

Alternatively, we implement the thermal vibrations as a much more accurate series expansion (from the mathematical tables by Stegun [32]), following the implementation in the BCA code MARLOWE [33]. This implementation also gives the zero-point vibrations. The implementation is provided as computer code in Table I. A comparison of the three calculation methods (Fig. 7) shows that at and above room temperature, all 3 models give very similar results. At temperatures well below room temperature, naturally the more accurate Stegun approximation or direct integral evaluations should be used.
TABLE I. Computer code (C language) segment giving the thermal displacement amplitude from a series expansion [32] of the Debye integral.

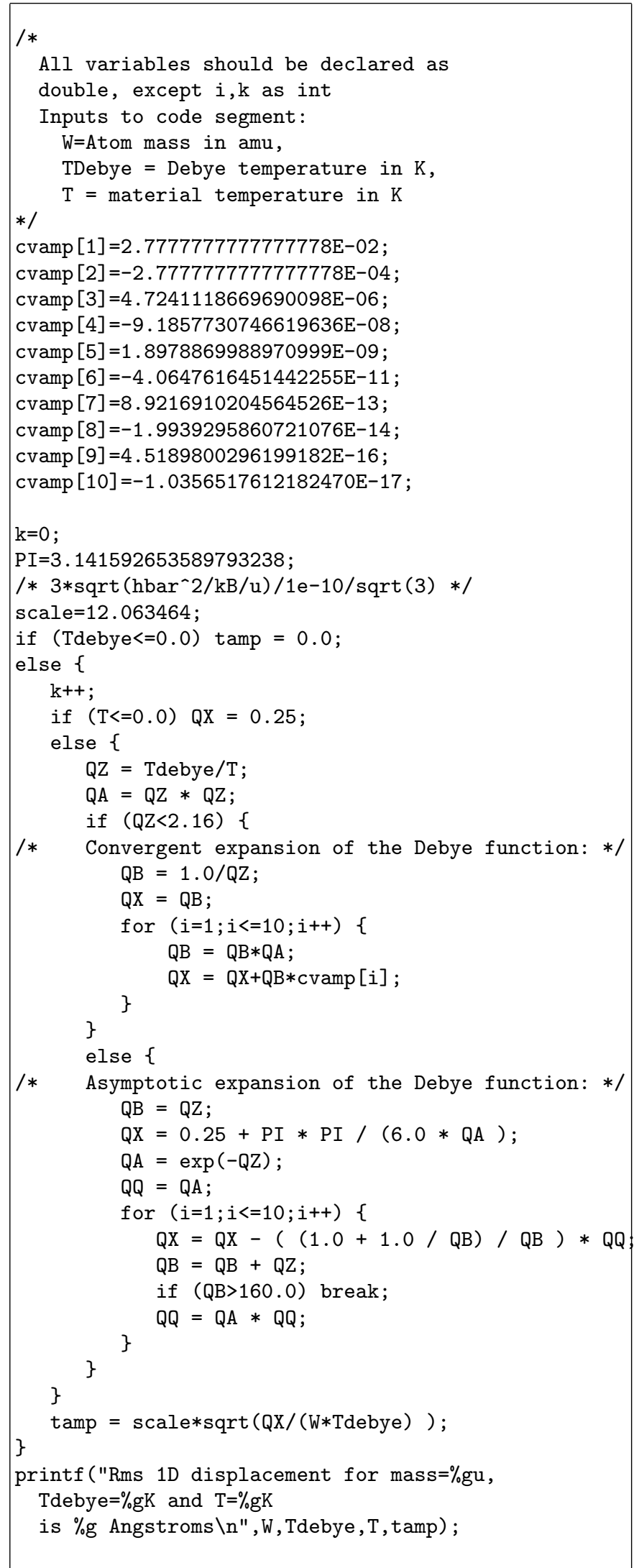




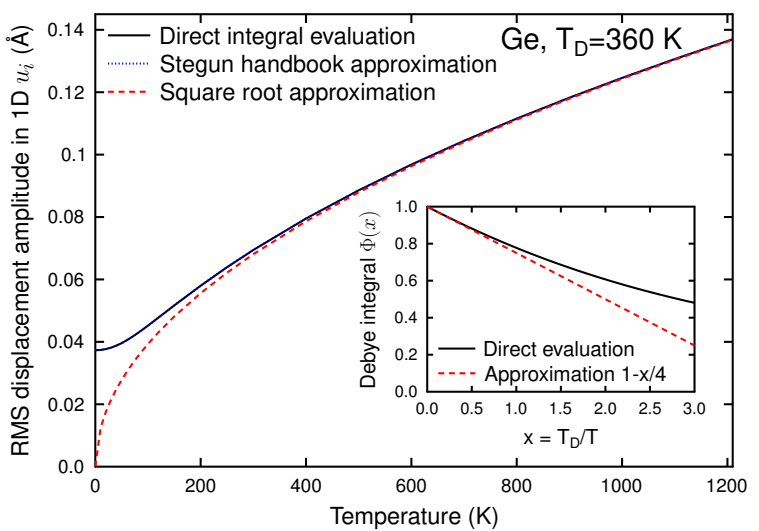

FIG. 7. (Color online) Comparison of the thermal displacement amplitudes obtained without any approximations (by highly accurate numerical evaluation of the Debye integral (8), with the series expansion implementation (Table I), as well as the high-temperature square root approximation (Eq. 12). The numerical evaluation and Stegun handbook approximation agree to such a high precision that these two curves are indistinguishable in the graph. The inset shows a comparison of direct evaluation of the Debye integral, Eq. (8) with the approximation Eq. (11).

In the simulations, the actual atom displacements are the obtained using

$$
x=x_{0}+u_{x} G[0,1]
$$

and correspondingly for $y$ and $z$. Here $G[0,1]$ denotes a Gaussian distributed random number with mean value 0 and standard deviation 1, generated using the standard Box-Muller method [26]. To ensure the random numbers do not start to repeat themselves during long simulations, the underlying uniform random numbers were generated with the Mersenne Twister [34] which has a repetition period of $2^{19937}-1$.

The random displacements were generated in this way for all atoms in the cells several times, generating 10 independent "initial state" cells with different displacements. After this, when new cell was generated in front of the moving ion, the atom coordinates are always picked from a randomly selected cell from among the 10 initial state cells.

As an example of the effect of the displacements on ion channeling, we simulated the implantation of $10 \mathrm{keV}$
Ge directly into the [110] channel in InP (with the Ge lattice constant, as in the main text). The results in Fig. 8 a) show that the range profiles do clearly depend on the temperature, with higher temperatures (larger displacements) closing the channel. On the other hand, the results in Fig. $8 \mathrm{~b}$ ) show that the results of the two different displacement models differ significantly only below $200 \mathrm{~K}$.

The results in the main text of the paper are obtained using displacements calculated with the square root ap-

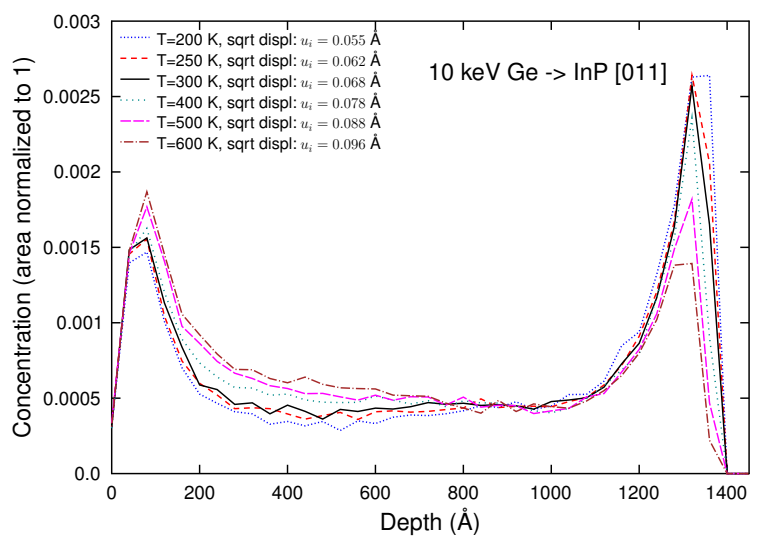

a)

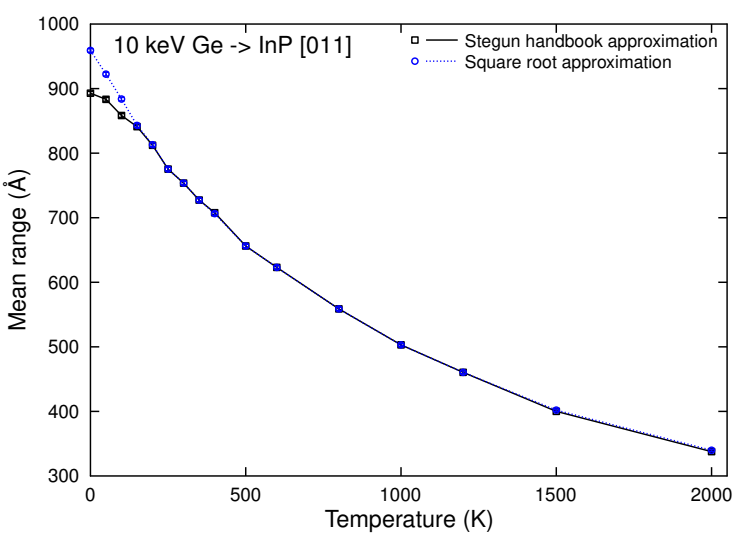

FIG. 8. (Color online) Comparison of the effect of thermal displacement amplitudes on ion ranges profiles during implantation in the [011] channel in InP. a) Effect on range profiles for the square root approximation displacements. b) Effect on mean range, comparing the highly accurate Stegun handbook approximation of the Debye integral with the square root approximation.

proximation (Eq. 12).
[1] M. T. Robinson and O. S. Oen, "The channeling of energetic atoms in crystal lattices," Appl. Phys. Lett. 2, 30 (1963).

[2] O. S. Oen and M. T. Robinson, "The effect of channeling on displacement cascade theory," Appl. Phys. Lett. 2,
83-85 (1963).

[3] E. V. Kornelsen, F. Brown, J. A. Davies, B. Domeij, and G. R. Piercy, "Penetration of Heavy Ions of keV Energies into Monocrystalline Tungsten," Phys. Rev. 136, 849 (1964). 
[4] L. T. Chadderton and F. H. Eisen, "On the transmission of Energetic protons through very thin crystals," Phil. Mag. 20, 195 (1969).

[5] L. C. Feldman, J. W. Mayer, and S. T. Picraux, Materials Analysis by Ion Channeling (Academic, New York, 1982).

[6] J. F. Ziegler and R. F. Lever, "Channeling of ions near the silicon (001) axis," Appl. Phys. Lett. 46, 358 (1985).

[7] J. Lindhard, "Influence of crystal lattice on motion of energetic charged particles," Kgl. Danske Vid. Selskab, Mat.- Fys. Medd. 34 (1965).

[8] G. Hobler, "Critical angles and low-energy limits to ion channeling in silicon," Radiat. Eff. Def. Sol. 139, 21-85 (1996).

[9] J. H. Barrett, "Monte Carlo Channeling Calculations," Phys. Rev. B 3, 1527-1547 (1971).

[10] P. J. M. Smulders and D. O. Boerma, "Computer Simulation of Channeling in Single Crystals," Nucl. Instr. Meth. B 29, 471-489 (1987).

[11] J. Sillanpää, K. Nordlund, and J. Keinonen, "Electronic stopping of Silicon from a 3D Charge Distribution," Phys. Rev. B 62, 3109 (2000).

[12] K. Nordlund, F. Djurabekova, and G. Hobler, "Large fraction of crystal directions leads to ion channeling," Phys. Rev. B 94, 214109 (2016).

[13] G. Greaves, J. A. Hinks, P. Busby, N. J. Mellors, A. Ilinov, A. Kuronen, K. Nordlund, and S. E. Donnelly, "Giant Sputtering Yields from Single-Ion Impacts on Gold Nanorods," Phys. Rev. Lett. 111, 065504 (2013).

[14] H. Holland-Moritz, A. Ilinov, F. Djurabekova, K. Nordlund, and C. Ronning, "Sputtering and redeposition of ion irradiated $\mathrm{Au}$ nanoparticle arrays: direct comparison of simulations to experiments," New J. Phys. 19, 13023 (2017).

[15] A. Turos, A. Stonert, B. Breeger, E. Wendler, W. Wesch, and R. Fromknecht, "Low temperature transformations of defects in GaAs and AlGaAs," Nucl. Instr. Meth. Phys. Res. 148, 401-405 (1999)

[16] E. Wendler, B. Breeger, and W. Wesch, "In situ investigation of AlAs/GaAs interfaces during ion implantation at $15 \mathrm{~K}, "$ Nucl. Instr. Meth. Phys. Res. B 175, 83-87 (2001).

[17] K. Lorenz, E. Alves, E. Wendler, O. Bilani, W. Wesch, and M. Hayes, "Damage formation and annealing at low temperatures in ion implanted ZnO," Appl. Phys. Lett. (2005), accepted for publication.

[18] E. Wendler and L. Wendler, "Empirical modeling of the cross section of damage formation in ion implanted III-V semiconductors," Appl. Phys. Lett. 100, 192108 (2012).

[19] T. S. Avila and P. F. P. Fichtner and A. Hentz and P. L. Grande, "On the use of MEIS cartography for the determination of Si 1-x Ge x thin-film strain," Thin Solid Films 611, 101-106 (2016).

[20] Yanwen Zhang, G. Malcolm Stocks, Ke Jin, Chenyang
Lu, Hongbin Bei, Brian C. Sales, Lumin Wang, Laurent K. Beland, Roger E. Stoller, German D. Samolyuk, Magdalena Caro, Alfredo Caro, and William J. Weber, "Influence of chemical disorder on energy dissipation and defect evolution in concentrated solid solution alloys," Nature communications 6 (2015).

[21] F. Granberg, K. Nordlund, M. W. Ullah, K. Jin, C. Lu, H. Bei, L. M. Wang, F. Djurabekova, W. J. Weber, , and Y. Zhang, "Mechanism of radiation damage reduction in equiatomic multicomponent single phase alloys," Phys. Rev. Lett. 116, 135504 (2016).

[22] K. Nordlund, "Molecular dynamics simulation of ion ranges in the $1-100 \mathrm{keV}$ energy range," Comput. Mater. Sci. 3, 448 (1995).

[23] J. Sillanpää, J. Peltola, K. Nordlund, J. Keinonen, and M. J. Puska, "Electronic stopping calculated using explicit phase shift factors," Phys. Rev. B 63, 134113 (2001).

[24] A presentation of the MDRANGE computer code is available on the World Wide Web in http://beam.acclab.helsinki.fi/ knordlun/mdh/mdh_program.html.

[25] J. Lindhard, M. Scharff, and H. E. Schiøtt, "Range concepts and heavy ion ranges," Kgl. Danske Vid. Selskab, Mat.- Fys. Medd. 33 (14), 1-42 (1963).

[26] W. H. Press, S. A. Teukolsky, W. T. Vetterling, and B. P. Flannery, Numerical Recipes in C; The Art of Scientific Computing, 2nd ed. (Cambridge University Press, New York, 1995).

[27] M. Blackman, Handbuch der Physik, Vol. VII (SpringerVerlag, Berlin, 1955).

[28] D. S. Gemmell, "Channeling and related effects in the motion of charged particles through crystals," Rev. Mod. Phys. 46, 129 (1974).

[29] M. Blackman, "The Specific Heat of Solids," in Handbuch der Physik, Vol. 7/1, edited by S. Flügge (Springer, Berlin, 1955) pp. 325-382.

[30] J. F. Ziegler, J. P. Biersack, and U. Littmark, The Stopping and Range of Ions in Matter (Pergamon, New York, 1985).

[31] N. W. Ashcroft and N. D. Mermin, Solid State Physics (Saunders College, Philadelphia, 1976).

[32] I. A. Stegun, Handbook of Mathematical Functions (National Bureau of Standards, Washington, DC, USA, 1964) p. 998

[33] M. T. Robinson and Ian M. Torrens, "Computer Simulation of atomic-displacement cascades in solids in the binary-collision approximation," Phys. Rev. B 9, 50085024 (1974).

[34] M. Matsumoto and T. Nishimura, "Mersenne twister: a 623-dimensionally equidistributed uniform pseudorandom number generator," ACM Transactions on Modeling and Computer Simulation 8, 3-30 (1998). 